\title{
Functional Excitatory Microcircuits in Neonatal Cortex Connect Thalamus and Layer 4
}

\author{
Cuiping Zhao, ${ }^{1,2}$ Joseph P. Y. Kao, ${ }^{3,4}$ and Patrick 0. Kanold ${ }^{1,2}$ \\ ${ }^{1}$ Department of Biology, Institute for Systems Research, and 2 Program in Neuroscience and Cognitive Science, University of Maryland, College Park, \\ Maryland 20742, and ${ }^{3}$ Medical Biotechnology Center, University of Maryland Biotechnology Institute, and ${ }^{4}$ Department of Physiology, University of \\ Maryland School of Medicine, Baltimore, Maryland 21201
}

The functional connectivity of the cerebral cortex is shaped by experience during development, especially during a critical period early in life. In the prenatal and neonatal cortex, transient neuronal circuits are formed by a population of subplate neurons (SPNs). However, SPNs are absent in the adult cortex. While SPNs are crucial for normal development of the cerebral cortex and of thalamocortical synapses, little is known about how they are integrated in the developing thalamocortical circuit. We therefore investigated SPNs in vitro in thalamocortical slices of A1 and medial geniculate nucleus (MGN) in mouse from postnatal day 1 (P1) to P13. We found that SPNs can fire action potentials at $\mathrm{P} 1$ and that their intrinsic membrane properties are mature after P5. We find that SPNs receive functional excitatory inputs from the MGN as early as P2. The MGN projections to SPNs strengthen between P2 and P13 and are capable of inducing action potentials in SPNs. Selective activation of SPNs by photostimulation produced EPSCs in layer 4 neurons, demonstrating a functional excitatory connection. Thus, SPNs are tightly integrated into the developing thalamocortical circuit and would be a reliable relay of early spontaneous and sound-evoked activity. The role of SPNs in development likely results from their strong excitatory projection to layer 4, which might function to regulate activity-dependent processes that enable mechanisms required for the functional maturation and plasticity of the developing cortex and thereby contribute to the development of normal cortical organization.

\section{Introduction}

Subplate neurons (SPNs) are among the earliest-appearing neurons in the cerebral cortex that are largely lost in the adult (Allendoerfer and Shatz, 1994; Kanold, 2004, 2009). Subplate neurons are located in the developing white matter of all cortical regions, including auditory cortex (ACX). In rodents, SPNs exist during the first 3 weeks of life, but some (15-39\%) remain into adulthood (Vandevelde et al., 1996; Price et al., 1997; Reep, 2000; Clancy et al., 2001; Torres-Reveron and Friedlander, 2007). Subplate death occurs earlier at caudal than rostral areas (Price et al., 1997), thus more SPNs might be present in ACX than in visual cortex at a given age. Removing SPNs in visual cortex, however, prevents the segregation and functional maturation of thalamocortical and intracortical projections, suggesting a key role for SPNs in cortical development (Ghosh and Shatz, 1992; Kanold et al., 2003; Kanold and Shatz, 2006). SPNs receive input from lower brain centers, such as the thalamus, before their axons grow to their targets in the cortex (Friauf et al., 1990; Allendoerfer and Shatz, 1994; Hanganu et al., 2002; Higashi et al., 2002; Molnár et al., 2003; Kanold, 2004). Glutamatergic SPNs project into the overlying developing cortical plate and are thought to excite cortical

\footnotetext{
Received Sept. 8, 2009; revised 0ct. 22, 2009; accepted 0ct. 31, 2009.

P.O.K. is supported by National Institutes of Health (NIH) Grant R01DC009607, and by the International Cerebral Palsy Research foundation; J.P.Y.K. is supported by NIH Grant R01 GM056481. We thank Dr. Karel Svoboda (Howard Hughes Medical Institute) for assistance in implementing LSPS and Julie Zemskova and Aminah Sheikh for histological assistance.

Correspondence should be addressed to Patrick 0. Kanold at the above address. E-mail: pkanold@umd.edu. D01:10.1523/JNEUROSCI.4471-09.2009

Copyright $\odot 2009$ Society for Neuroscience $\quad 0270-6474 / 09 / 2915479-10 \$ 15.00 / 0$
}

neurons (Friauf et al., 1990; Finney et al., 1998; Piñon et al., 2009). Such an excitatory function of SPNs on layer 4 neurons combined with reliable transmission of thalamic activity through SPNs could control activity-dependent processes in layer 4 leading to mature cortical circuitry (Kanold et al., 2003; Kanold and Shatz, 2006; Kanold, 2009). However, direct evidence of such an excitatory connection has been lacking.

While SPNs are present before and during the onset of functional hearing [ postnatal day (P)8-P9] (Alford and Ruben, 1963; Mikaelian and Ruben, 1965; Mikaelian et al., 1965; Shnerson et al., 1983; Friauf et al., 1990; Romand and Ehret, 1990; Catalano et al., 1991; Friauf and Shatz, 1991; Ehret and Romand, 1992; Molnár and Blakemore, 1995), little is known about the functional properties of SPNs in ACX. Given the potential key role of SPNs mediating thalamic excitation to the cortical plate we therefore investigated SPNs and their associated circuits by in vitro recordings in thalamocortical brain slices from neonatal mouse ACX.

We found that SPNs fire action potentials as early as P1. SPNs receive thalamic (MGN) inputs as early as tested (P2) and over postnatal development the number of MGN inputs to SPNs increases. MGN inputs can elicit action potentials in SPNs, aided by the high input resistance and depolarized resting potential of SPNs at young ages. In addition, we find that selective SPN stimulation results in EPSCs in layer 4 neurons, thus SPNs provide excitatory input to layer 4 . Together, we demonstrate that SPNs are integrated into the developing thalamocortical microcircuit, receive functionally strong thalamic input, and provide a strong excitatory feedforward excitation to developing layer 4, the target 

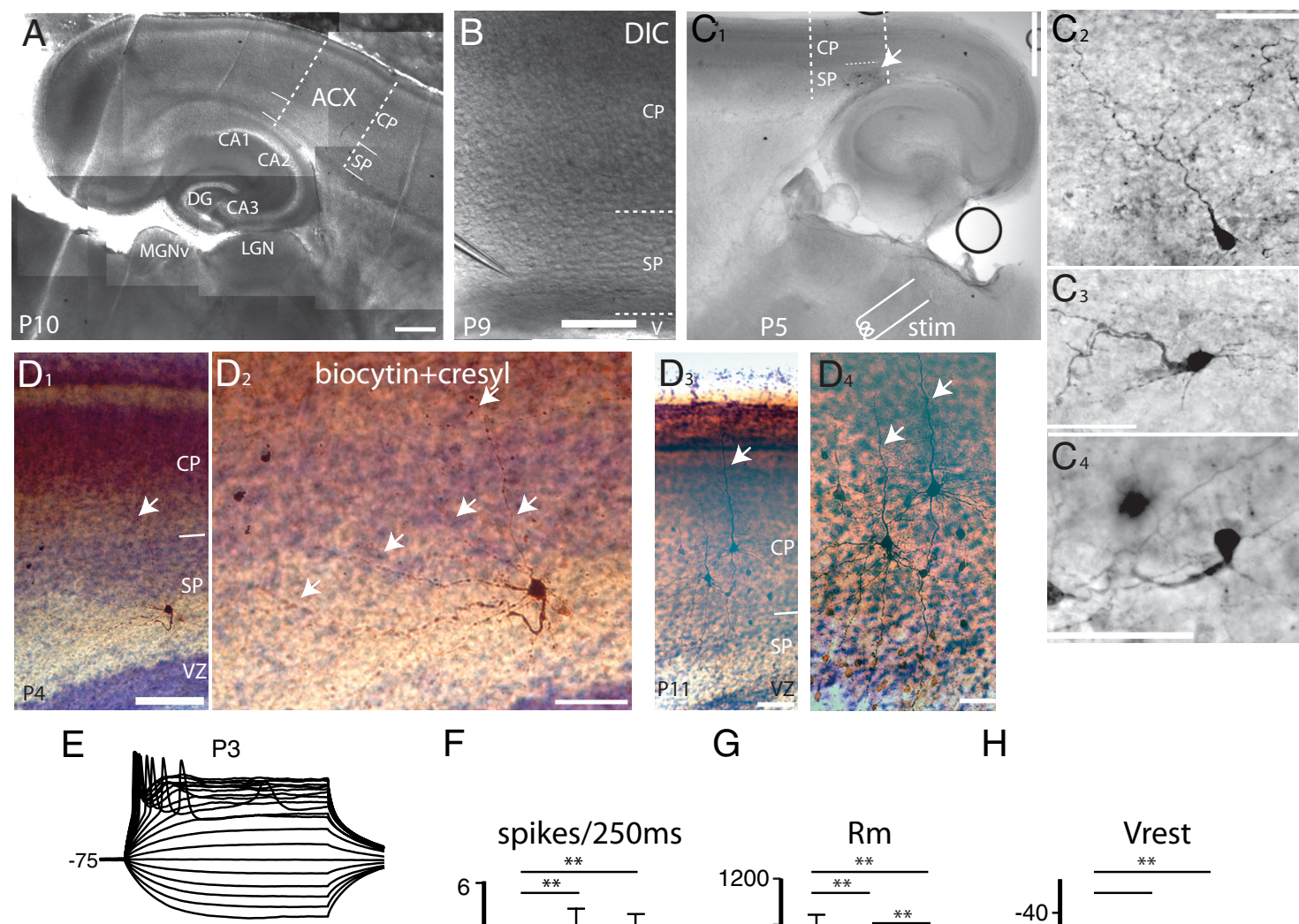

$\mathrm{F}$

G

$\mathrm{H}$
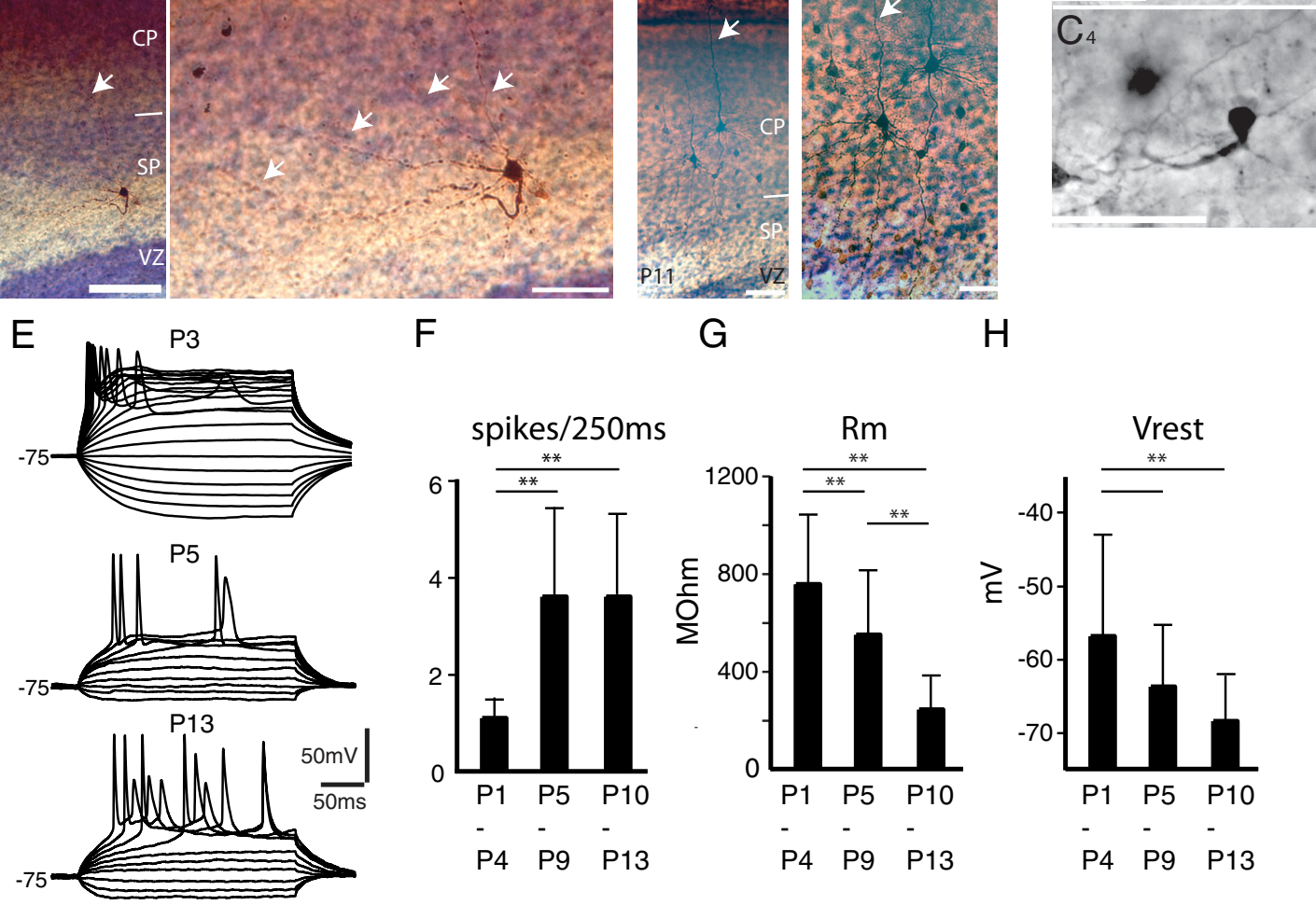

Figure 1. Intrinsic properties of SPNs in auditory cortex. $A$, Thalamocortical slice under DIC at P10. MGN and ACX are indicated. Scale bar, $500 \mu \mathrm{m}$. B, Higher-power picture during a recording from a SPN at P9. Note the clear differentiation of the subplate zone (SP) and cortical plate (CP) under DIC. The subplate appears horizontally organized while layer 6 appears darker. Scale bar, $200 \mu \mathrm{m}$. $\mathrm{V}$ indicates ventricle. C, P5 thalamocortical slice after recording from multiple cells and subsequent biocytin staining. Stimulation site in MGN is indicated ("stim"). Note the stained cells in the subplate in ACX (arrowhead). High-power pictures show three of the stained subplate neurons, illustrating diverse morphology. Scale bars: $500 \mu \mathrm{m}$ (slice), $50 \mu \mathrm{m}$ (cells). D, Subplate neurons and layer 6 neurons show different morphologies. Shown are biocytin stained neurons in a $600 \mu \mathrm{m}$ slice counterstained with cresyl violet. The subplate zone (SP) is readily distinguished from the cortical plate in cresyl stains as being located below the transition between radial oriented cells and horizontally oriented cell bodies (future layer 6B). D1 and D2 show subplate neuron. The cell body is located in the subplate and axons project onto the cortical plate while dendrites are in subplate and layer 6 . VZ indicates the densely stained ventricular zone. Scale bars: $100 \mu \mathrm{m}$ (D1), $50 \mu \mathrm{m}$ (D2). D3 and D4 show pyramidal cells in layers 5/6. Note the prominent apical dendrites (arrows) that reach into superficial cortical layers. Scale bars: $100 \mu \mathrm{m}$ (D3), $50 \mu \mathrm{m}$ (D4). Due to the thickness of the section drying is uneven thus shrinkage of layer $2 / 3$ is more pronounced. $\boldsymbol{E}$, Current injections cause action potentials at all ages studied. Shown are traces evoked with various current injections at 3 ages. Cells were held at $-75 \mathrm{mV}$. $\boldsymbol{F}$, Average numbers of action potentials during $250 \mathrm{~ms}$ current injections. Note that the numbers of action potentials increase between P1/4 and P5/9. $\boldsymbol{G}, \boldsymbol{H}$, Input resistance $\left(R_{\mathrm{m}}\right)$ and resting potential $\left(V_{\text {rest }}\right)$ determined from linear fits to $I-V$ curve below action potential threshold. Both $R_{\mathrm{m}}$ and $V_{\text {rest }}$ decrease during the postnatal period. ${ }^{* *} p<0.05$.

layer of thalamic axons. This feedforward excitatory circuit might influence maturational processes in layer 4 , leading to mature thalamocortical and intracortical connectivity.

\section{Materials and Methods}

Slice physiology and imaging methods are as published previously (Kanold et al., 2003; Kanold and Shatz, 2006). All procedures followed the University of Maryland College Park animal use regulations.

Slice preparation. Mice (C57BL/6) from Jackson (jax.org) are deeply anesthetized with isofluorane (Halocarbon). A block of brain containing ACX and MGN is removed and thalamocortical slices (500-600 $\mu \mathrm{m}$ thick) are cut on a vibrating microtome (Leica) in ice-cold ACSF containing (in mM): $130 \mathrm{NaCl}, 3 \mathrm{KCl}, 1.25 \mathrm{KH}_{2} \mathrm{PO}_{4}, 20 \mathrm{NaHCO}_{3}, 10$ glucose,
1.3 $\mathrm{MgSO}_{4}, 2.5 \mathrm{CaCl}_{2}$ (pH 7.35-7.4, in $95 \% \mathrm{O}_{2}-5 \% \mathrm{CO}_{2}$ ). The cutting angle is $\sim 15$ degrees from the horizontal plane (lateral raised) at older ages (Cruikshank et al., 2002) and somewhat steeper at younger ages to compensate for the lateral growth of cortex. Slices are incubated for $1 \mathrm{~h}$ in ACSF at $30^{\circ} \mathrm{C}$ and then kept at room temperature. For recording, slices are held in a chamber on a fixed stage microscope (Nikon FN1) and superfused $(2-4 \mathrm{ml} / \mathrm{min})$ with ACSF at room temperature. The location of the recording site in ACX was identified by landmarks (location of hippocampus) (see Fig. 1A) and validated by monosynaptic responses to electrical MGN stimulation.

Identification of subplate. The subplate zone is readily identified as a relative cell sparse area between the large layer 6 pyramidal cells and the ventricular zone. The subplate encompasses horizontally oriented cells in 
A
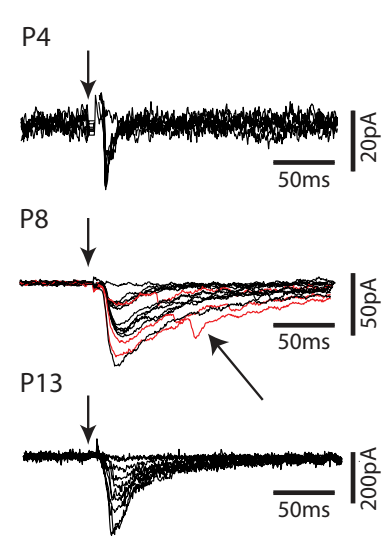

C

minimal $(\mathrm{pA})$ maximal $(\mathrm{pA})$

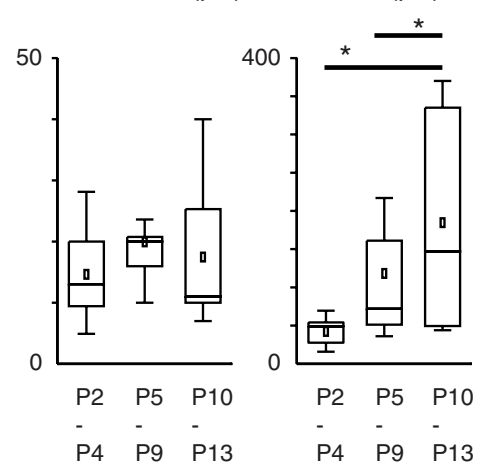

EPSC amplitude (pA)
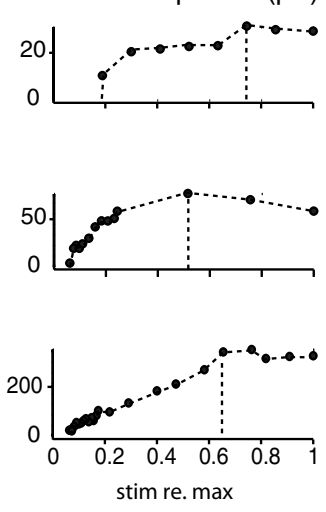

stim re. $\max$
B

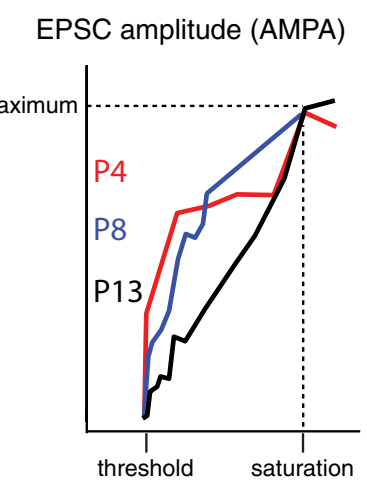

D latency (ms)

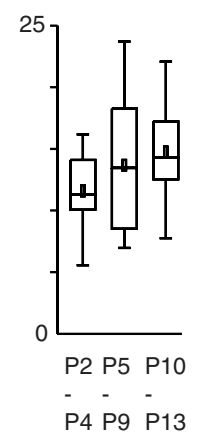

Figure 2. Excitatory MGN inputs to SPNs throughout the postnatal period. $\boldsymbol{A}$, MGN evoked EPSCS with increasing stimulation currents at 3 ages. Traces are shown on the left. Peak EPSC amplitudes as functions of stimulus strength (I0-curve) are shown on right. For comparison, stimulus strength was normalized to the maximum stimulus strength used in a particular cell. Note the limited dynamic range of EPSC amplitudes at young ages. Dashed lines indicate where saturation was reached. Note the occasional disynaptic responses (red traces). $\boldsymbol{B}$, Graph shows a superposition of the 10 curves in $\boldsymbol{A}$ normalized to maximum EPSC and saturation stimulus level. Note the steeper increase in the 10 curve from threshold to saturation at younger ages. C, Quantification of EPSC amplitudes. Box plots show EPSC amplitude at threshold (minimal reliably evoked EPSC), maximal evoked EPSC, and the ratio of these values. Box plots show median (line), mean (square), and the $25 \%$ and $75 \%$ quartiles. The threshold EPSC amplitude does not change (P1/4: 14.6 $\pm 7.2 \mathrm{pAN}=9 ; \mathrm{P} 5 / 9: 19.9 \pm 8 \mathrm{pAN}=12 ; \mathrm{P} 10 / 13: 17.45 \pm 11.8 \mathrm{pAN}=8)$, while maximal EPSC amplitude (P1/4: $42.4 \pm 18.1 \mathrm{pA} ; \mathrm{P5} / 9: 121 \pm 105 \mathrm{pA} ; \mathrm{P} 10 / 13: 184 \pm 149 \mathrm{pA})$ and the ratio (P1/4: $3.1 \pm 1.2 ; \mathrm{P} 5 / 9: 4.3 \pm 5.6 ; \mathrm{P} 10 / 13$ : $10.1 \pm 6.5$ ) increase from P2/4 to P5/9 and P10/13 ( ${ }^{*} p<0.05$ ). D, Latencies of the monosynaptic EPSC (time to peak). Box plots show median (line), mean (square) and the $25 \%$ and $75 \%$ quartiles. Peak latencies were similar at all ages (P1/4: $11.6 \pm 3.2 \mathrm{ms,}$ P5/9: $13.66 \pm 5.76 \mathrm{~ms}$, P10/13: $14.74 \pm 4.32 \mathrm{~ms}$, all $p>0.1)$.

an upper sublamina below layer 6 (future layer $6 \mathrm{~b}$ ) and deeper more scattered neurons in the future white matter. The upper sublamina is separated from layer 6 by a cell sparse zone. Compared to other neurons, the deeper subplate neurons in young rodents stain relatively poorly for markers such as NeuN but are apparent under differential interference contrast (DIC) where subplate neurons intermingled with horizontally traveling fiber can be readily seen due to their horizontal appearance (see Figs. $1 A, B, 6$ ).

Electrophysiology. Whole-cell recordings are performed with a patchclamp amplifier (Multiclamp 700B, Molecular Devices) using pipettes with input resistance of $4-8 \mathrm{M} \Omega$. Data are acquired with a Digidata $A D$ board (Molecular Devices) under pClamp (v9 and v10) and analyzed offline using MATLAB (MathWorks). Electrodes are filled with (in mM) $110 \mathrm{~K}$-gluconate, $4 \mathrm{KCl}, 4 \mathrm{NaCl}, 0.2 \mathrm{CaCl}_{2}, 10 \mathrm{HEPES}, 1.1 \mathrm{EGTA}, 2$ Mg-ATP, $1 \mathrm{MgCl}_{2}$ and 5 glutathione ( $\mathrm{pH} 7.2,300 \mathrm{mOsm}$ ). Membrane voltages are corrected for an estimated liquid junction potential of 14 $\mathrm{mV}$. Some cells were recorded using a solution containing $115 \mathrm{~mm}$ cesium methanesulfonate $\left(\mathrm{CsMeSO}_{3}\right) 5 \mathrm{NaF}, 10$ EGTA, 10 HEPES, 15 $\mathrm{CsCl}, 3.5 \mathrm{MgATP}, 3 \mathrm{QX}-314$ (pH 7.25, $300 \mathrm{mOsm}$ ). We found no obvious differences in EPSC sizes with these two solutions. For LSPS all cells were recorded with this solution. Biocytin or neurobiotin $(0.5 \%)$ is added to the electrode solution as needed. Electrical stimulation: We use a bipolar electrode (Microprobe, biphasic, $0.2 \mathrm{~ms}$, at $0.03 \mathrm{~Hz}$ ) coupled to a stimulus isolator (Cygnus). Photostimulation: $0.5-1 \mathrm{~mm}$ caged glutamate $(N-$ (6-nitro-7coumarylmethyl)-L-glutamate; Ncm-Glu) (Kao, 2006) is added to the ACSF. Without UV light, this compound has no effect on neuronal activity $(N=40$ neurons) (Kao, 2006). UV laser light $(500 \mathrm{~mW}, 355 \mathrm{~nm}, 100 \mathrm{kHz}$ repetition rate, DPSS, $2-10 \mathrm{~ms}$ pulses) is split via a $33 \%$ beam splitter (CVI Melles Griot) and coupled via a fiber optical coupler (Oz Optics) into an optical fiber ( $25 \mu \mathrm{m}$ core diameter, Oz Optics). The distal end of the fiber is held within a bent glass pipette to reduce beam elongation and apply focused light to a small area. We visualized the stimulated site for all cells by taking a picture of the light beam to ensure that stimulation was limited to the subplate. In preliminary experiments we varied laser power and determined that this power provided reliable direct activation of neurons recorded in wholecell patch configuration. For laser-scanning photostimulation (LSPS), the other fraction of the split beam is coupled into a microscope via scan mirrors (Cambridge Technology) and a dichroic mirror (Shepherd et al., 2003). The laser beam in LSPS enters the slice axially through the objective (Olympus $4 \times, 0.16 \mathrm{NA}$ / air) and has a diameter of $\sim 15 \mu \mathrm{m}$. Laser power at the sample is $<20 \mathrm{~mW}$. In preliminary experiments we varied laser power and determined that this power provided reliable activation of neurons. We typically imaged $16 \times 16$ or $32 \times 32$ stimulation sites spaced $20-50 \mu \mathrm{m}$ enabling us to image areas of $1 \mathrm{mmx} 1 \mathrm{~mm}$. Stimuli are applied at $0.5-1 \mathrm{~Hz}$. Data acquisition is performed by National Instruments $\mathrm{AD}$ boards and custom software (Ephus) (Shepherd et al., 2003). Ephus (available online at http://research.janelia.org/labs/display/ ephus/Ephus) is written in MATLAB and adapted to our setup. Analysis was performed similar to methods described previously (Shepherd et al., 2003) by using custom software written in MATLAB. To detect monosynaptically evoked EPSCs we detected peak EPSC amplitudes in a $\sim 60 \mathrm{~ms}$ time window after the stimulation. Traces containing a short-latency $(<15-20 \mathrm{~ms}$ "direct") response were discarded from the analysis (see Fig. 6 , black patches) as were traces that contained longer latency inward currents of long duration (>100 ms). (Fig. 6, gray patches) These currents could be sometimes seen in locations surrounding $(<50 \mu \mathrm{m})$ areas that gave a direct response. Occasionally, some of the direct responses contained synaptically evoked responses that we did not separate out, leading to an underestimation of local short-range connections. Cells that only showed direct responses were excluded from the analysis.

Drugs. We use NBQX $(50 \mu \mathrm{M})$ to block AMPA currents and TTX (1 $\mu \mathrm{M})$ to block action potentials. All chemicals and drugs were obtained from Sigma.

Statistics. Results are plotted as means \pm SD, compared with a Students $t$ test, and deemed significant if $p<0.05$.

\section{Results}

We investigated the physiological characteristics of subplate neurons in mouse auditory cortex over three age ranges (P1-4, P5$\mathrm{P} 9$, and P10-P13), approximately corresponding to distinct functional states of the auditory system. Hair cells do not become functional before P5 and thus only spontaneous evoked activity is present in the auditory system. After onset of hair cell function 
but before the opening of the ear canal, spontaneous and high-threshold soundevoked responses might occur. In mice of the C57BL/6 strain the ear canal opens at P9 (Shnerson and Pujol, 1983; Pujol et al., 1997; Ruebsamen and Lippe, 1997). After ear opening, low-threshold hearing develops, followed by an early critical period ( $\sim$ P11-13 in rat) (de Villers-Sidani et al., 2007).

\section{The intrinsic properties of subplate neurons mature over the postnatal period}

We recorded from SPNs $(N=140)$ in thalamocortical slices of A1 over the first 2 postnatal weeks using whole-cell patchclamp recordings (Fig. $1 A-C$ ). SPNs are located in the future white matter below layer 6 and have diverse morphology (Allendoerfer and Shatz, 1994; Luhmann et al., 2000) (Fig. 1B-D). In response to current injections, SPNs at all ages were able to fire action potentials (APs) (Fig. $1 E)$. However, in response to depolarization, the pattern of APs and the number of spikes evoked varied strongly with age. At early ages (P1/4) sustained current injection resulted in only one AP in 11 of 13 SP neurons, while this firing pattern was rare at ages older than P5 (4/37 cells). Figure $1 F$ shows that maximal number of evoked action potentials to a $250 \mathrm{~ms}$ depolarization increased from $\mathrm{P} 1 / 4$ to $\mathrm{P} 10 / 13$ (P1/4: $1.1 \pm 0.3, \mathrm{P} 5 / 9: 3.6 \pm 1.8, \mathrm{P} 10 / 13: 3.6 \pm$ 1.6 , both $p<0.01)$. However, action potential threshold remained constant over this age range $(-48.4 \pm 6 \mathrm{mV}$ vs $-50.9 \pm 4 \mathrm{mV}, p>0.1)$.

One feature of SPNs at P1/4 is a sustained depolarization after the AP (Fig. $1 E$ ), which might be due to low expression levels of $\mathrm{K}$-channels. To test the availability of K-channels, we measured the membrane resistance $\left(R_{\mathrm{m}}\right)$ of SPNs by linear fits to the $I-V$ curve below spike threshold and found that $R_{\mathrm{m}}$ decreased between P1/4 and P10/13 (P1/4: $757 \pm 284 \mathrm{M} \Omega, \mathrm{P} 5 / 9: 554 \pm 260$ $\mathrm{M} \Omega, \mathrm{P} 10 / 13: 285 \pm 95 \mathrm{M} \Omega$, both $p<0.05$ ) (Fig. $1 G$ ). In addition, in the first 2 postnatal weeks, the resting membrane potential showed a steady hyperpolarizing trend $(\mathrm{P} 1 / 4:-56.9 \pm 13.9 \mathrm{mV}$, P5/9: $-63.7 \pm 8.4 \mathrm{mV}, \mathrm{P} 10 / 13:-68.5 \pm 6.3 \mathrm{mV}$; P1/4 to P5/8 $p=0.13 ; \mathrm{P} 10 / 13 p<0.05)($ Fig. $1 H)$. These results also indicate that SPNs at early ages require less depolarization $(8.5 \mathrm{mV}$ at P1/4 vs $17.6 \mathrm{mV}$ at $\mathrm{P} 10 / 13$ ) to trigger an action potential-that is, SPNs at early ages can more easily respond to synaptic inputs.

\section{SPNs receive functionally strong thalamic inputs}

SPNs are thought to receive thalamic inputs, as has been demonstrated directly in the cat visual and rodent somatosensory cortex by CSD analysis and optical recordings (Friauf et al., 1990; Allendoerfer and Shatz, 1994; Hanganu et al., 2002; Higashi et al., 2002; Molnár et al., 2003). We therefore investigated if SPNs in ACX receive inputs from the auditory thalamus [medial geniculate nucleus (MGN)] and how these inputs change over the course of development. MGN fibers reach the cortical plate before birth (Gurung and Fritzsch, 2004). Electrical stimulation of the MGN
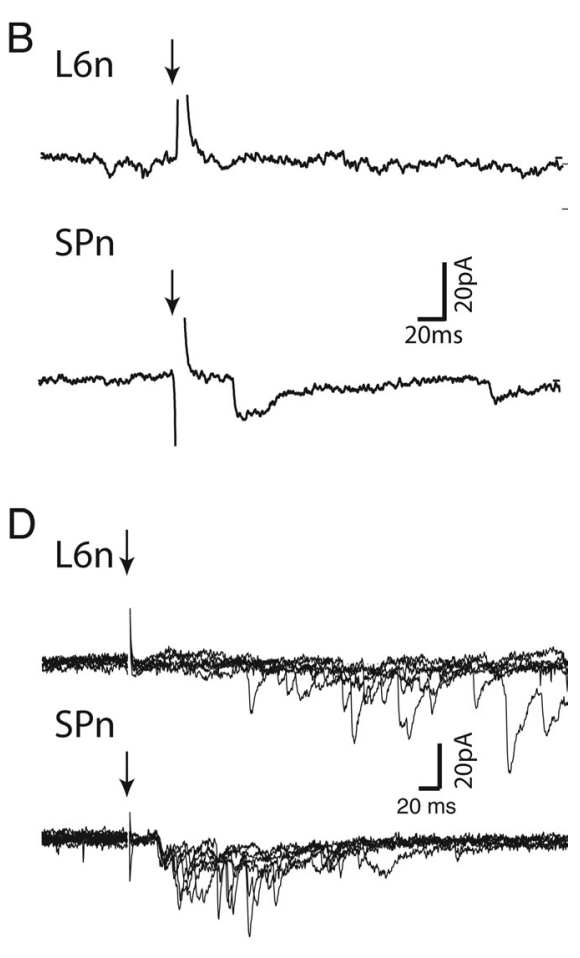

$\mathrm{D}$
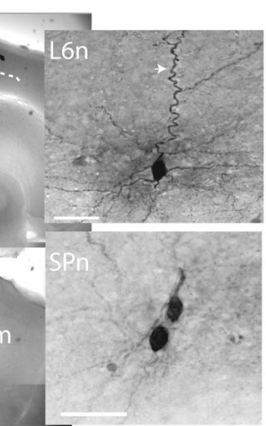

Figure 3. MGN selectively activates SPNs. A, Picture of a slice ( $600 \mu \mathrm{m}$ thick) from a P5 animal in which both layer 6 and SPns

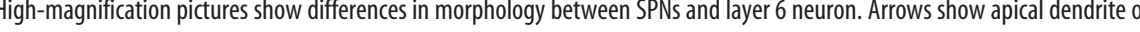
er 6 neurons traveling to superficial layers. Scale bars: $1 \mathrm{~mm}, 50 \mu \mathrm{m}$. D. Traces from SPN and layer 6 neuron in C to electrical stimulation of MGN (multiple traces superimposed). Note the reliable short latency EPSC in the SPN while the layer 6 neuron showed long latency EPSCs on some trials that started when SPNs ceased to receive inputs.

as early as P2 resulted in short-latency EPSCs in SPNs (Fig. 2A), indicating that monosynaptic transmission from thalamus to cortex is already present before the onset of hearing. Occasionally, we observed larger, long-latency EPSCs, which might reflect disynaptic inputs (Fig. $2 A$, red traces). We focused our analysis on the monosynaptically evoked EPSC, which was taken as either the only evoked EPSC, or the first (short-latency) EPSC if multiple EPSCs were observed.

Increasing stimulation amplitudes resulted in corresponding increases in EPSC amplitude (Fig. $2 \mathrm{~A}$ ). In some cells, especially at young ages, these increases occurred in a stepwise manner (Fig. $2 A$, top), suggesting the activation of separate synapses, whereas at older ages such steps were less clear (Fig. $2 A$, bottom, $B$ ). The minimally evoked EPSC amplitude was similar at P1/4 and P10/ 13, while the maximally evoked EPSCs were larger at older ages (Fig. 2C). The ratio of maximal to minimal EPSC is an estimate of the number of thalamic fibers innervating SPNs, and the increased ratio (Fig. $2 C$ ) suggests that the thalamo-subplate synaptic connection does not prune but increases its numbers of connections during the postnatal period, even though SPNs are starting to disappear. EPSC latencies (time to peak) were similar at all ages (Fig. 2D).

However, we did not observe any MGN stimulation evoked outward currents under our recording conditions at any age, which suggests that MGN inputs might not engage inhibitory circuits synapsing on SPNs. 
A
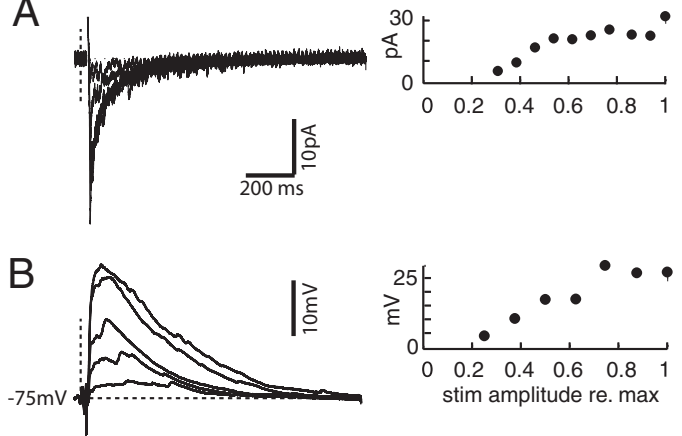

C

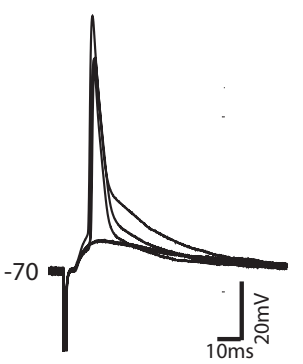

D

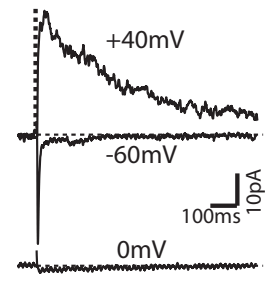

Figure 4. MGN inputs can cause action potentials in SPNs due to long lasting depolarization. $A$, SPN at P7. MGN stimulation at increasing intensities results in EPSCs of increasing amplitude. Selected traces (left) and peak EPSC amplitudes and each stimulus level (normalized to the saturating level) (right). $\boldsymbol{B}$, MGN stimulation at increasing intensities results in EPSPs of increasing amplitude (same cell as in $\boldsymbol{A}$ ). Selected traces (left) and peak EPSP amplitudes and each stimulus level (normalized to the saturating level) (right). Note the large maximal depolarization. Cell was held at negative potentials to prevent spiking. C, Another cell. MGN stimulation is able to evoke action potentials. D, MGN stimulation causes both AMPA and NMDA currents in SPNs. Shown is a SPN at P4. The electrode solution contained QX314 to block Na-currents. Currents were separated by holding SPNs at $-60 \mathrm{mV}$ or $+40 \mathrm{mV}$. Trace below shows that no evoked current reversed at $0 \mathrm{mV}$, suggesting that the synapse is glutamatergic. Note that no outward currents were observed, thus MGN stimulation did not recruit GABAergic neurons.

Since layer 6 neurons are located close to SPNs, we investigated if layer 6 neurons receive MGN inputs at these ages. We recorded from layer 6 neurons $(N=8)$ (Figs. $1 D, 3 A, C)$ in the same slice in which SPN showed MGN inputs (Fig. $3 B, D$ ). In these slices and by stimulating at the same stimulation site, layer 6 cells did not show short latency inputs (Fig. $3 B, D$ ). However, 2/8 layer 6 neurons showed long latency inputs (Fig. 3D). Comparison of the layer 6 responses with SPN responses to stimulation at the same site (Fig. 3D) shows the large differences in latencies. These data might also indicate that SPNs receive MGN inputs and drive cortical circuits that give rise to layer 6 inputs.

The high input resistance at young ages suggests that even small EPSCs might have a large effect on the membrane potential of SPNs. Given the relatively depolarized resting potential and the smaller depolarization required to reach action potential threshold, it is possible that small EPSCs might produce large EPSPs and action potentials. Figure $4, A$ and $B$, shows sequential voltage and current-clamp recordings in a cell at P7 using stimuli of identical intensities. Note that even at the lowest intensities, which produced only a small EPSC (Fig. 4A), a large, long-lasting EPSP was generated (Fig. $4 B$ ) (cell held hyperpolarized to prevent action potentials). If the cells were held close to their normal resting potentials (Fig. 1), these large EPSPs were able to produce action potentials $(N=3$ cells) (Fig. $4 C)$. These large and long-lasting EPSPs might partially be due to the high input resistance and long time constant of SPNs, but also could indicate recruitment of NMDA receptors by sufficient depolarization. We therefore investigated if NMDA receptors are present at the MGN-SPN synapse by holding SPNs at two different potentials, $-60 \mathrm{mV}$ and $+40 \mathrm{mV}$. Holding SPNs between P4 and P13 at depolarized potentials $(+40 \mathrm{mV})$ revealed a large long lasting outward current in $15 / 15$ SPNs tested, which was abolished by holding the neuron at $\sim 0$ $\mathrm{mV}$ (the reversal potential of glutamate receptors) (Fig. $4 D$ ). This indicates that this current was carried by glutamate. In addition, the lack of inward currents at $0 \mathrm{mV}$ indicates that MGN stimulation did not recruit GABAergic inputs to SPNs. The conductance ratio of the NMDA and AMPA component of the glutamatergic current $g_{\text {NMDA }} / g_{\text {AMPA }}$ (calculated from the peak currents by dividing by the driving force) was $3.4 \pm 1.5$. Together, these data show that synaptic transmission from MGN to SPN is reliable and functionally strong at young ages, and includes both AMPA and NMDA receptor-mediated components. Thus, SPNs can reliably relay MGN activity to the developing cortex.

\section{Subplate neurons excite layer 4 neurons}

SPNs axons project into the developing cortical plate, especially radially to layer 4 (Friauf et al., 1990; Allendoerfer and Shatz, 1994; Piñon et al., 2009). One outcome of SPN removal is the lack of maturational processes that are thought to depend on neuronal activity, such as the upregulation of KCC2 (Kanold et al., 2003; Kanold and Shatz, 2006). Thus, the strong maturational effect of SPNs on layer 4 might be mediated by strong excitatory projections from SPNs to layer 4. To investigate the functional effect of SPNs on layer 4, SPNs need to be selectively stimulated. However, common techniques such as electrical stimulation of the white matter will excite both SPNs and thalamic axons. We therefore selectively activated SPNs by photostimulation. Selective stimulation of SPNs was achieved by bathing the slice in caged glutamate solution and locating an optical fiber tip in the subplate (Fig. 5A). The illuminated area has an elliptical shape, with the long axis being $<100 \mu \mathrm{m}$ and the short axis being $<50$ $\mu \mathrm{m}$, and is entirely contained in the subplate. We verified that the activation area is in the subplate by visualizing the beam (Fig. 5A). In the presence of caged glutamate in the bath a brief laser pulse led to short-latency inward currents in SPNs, but only if the SPN was in the center of the illuminated area (Fig. $5 B$, black trace) $(N=5)$. These photostimulated inward currents were readily blocked by NBQX and were thus glutamatergic (Fig. 5B, red trace). Current-clamp recordings showed that these currents were sufficient to evoke action potentials in SPNs at all ages studied (Fig. $5 C, D$ ). The latency of the evoked action potential was consistent across trials (Fig. $5 E$ ). We then investigated whether photostimulation of SPNs evokes synaptic currents in layer 4 cells located radially above SPNs. Following photostimulation of SPNs, layer 4 cells (30/32) showed an inward current (Fig. 5F, black trace), while layer $2 / 3$ cells $(2 / 2)$ did not show any evoked currents following SPN stimulation. The inward current in layer 4 cells was blocked by TTX (Fig. $5 F$, red trace), indicating that neuronal spiking, presumably from SPNs, was involved, and that the current did not originate by direct activation of glutamate receptors on the layer 4 neuron by the uncaged glutamate. The onset latency of the photostimulation-evoked EPSC matched the latency of SPN firing (Fig. 5F), and was longer than the inward currents produced by direct activation of the SPNs, further suggesting that it was generated by SPN activation. Following SPN stimulation, EPSC amplitudes in layer 4 ranged from 10 to 170 pA (Fig. 5G). However, the average amplitude did not change over development (P5/8: $34 \pm 29.3 \mathrm{pA} N=12$, P9/13: $48.9 \pm 52$ pA $N=15 ; p>0.1)$. Because input resistances of layer 4 cells ranged from 310 to $1184 \mathrm{M} \Omega$ (mean $717 \pm 255 \mathrm{M} \Omega$ ), these EPSCs should strongly depolarize layer 4 neurons, just as 
MGN inputs depolarize SPNs. Indeed, current-clamp recordings ( $N=3$ cells) revealed large EPSPs in layer 4 following photostimulation of SPNs (Fig. $5 \mathrm{H}$ ), indicating a strong functional impact of SPNs on layer 4 neurons. Together, these data indicate a strong functional excitatory connection between SPNs and layer 4 neurons.

We next investigated the spatial pattern of SPN inputs to layer 4 by using laser-scanning photostimulation (Shepherd et al., 2003). The laser beam was targeted on a $16 \times 16$ to $32 \times 32$ pixel array covering an area of the slice (Fig. 6A). Cellattached patch recordings from SPNs $(N=3)$ showed that laser pulses were able to trigger short latency action potentials in SPNs (Fig. $6 B, C$ ). Plotting the spatial location of stimulation sites that cause SPN spiking showed that photostimulation had to be performed within $\sim 100 \mu \mathrm{m}$ of the SPN soma (Fig. 6B,C). Plotting the latency to the first spike (Fig. $6 C$ ) shows that activation close to the soma caused spikes with the shortest latency. Thus, only photostimulation of the soma or proximal dendrites is sufficient to activate SPNs.

We next investigated the origin of synaptic inputs to layer 4 by whole-cell recordings from layer 4 neurons during LSPS $(N=21)$. Photostimulation caused large amplitude short latency inward currents (labeled direct in Fig. 6D1), which reflect the direct activation of the cell under study (Shepherd et al., 2003; Barbour and Callaway, 2008). We also observed smaller amplitude long latency inward currents (labeled "evoked" in Fig. 6D1). These currents likely reflect EPSCs evoked by photostimulation of presynaptic cells. Plotting the amplitude of the pEPSC as function of stimulation location showed that direct responses (Fig. 6D2, pink boxes) were clustered around the cell body (Fig. 6D2, red circle). Evoked responses were also clustered but in a spatially distinct region (Fig. 6D2, yellow boxes). To identify the laminar source of the evoked response, we plotted the amplitude of the responses and overlayed this graph with the DIC image of the slice (Fig. $6 D 3)$. We find that the source of the excitatory inputs was located the subplate but not layer 6 (Fig. 6D3). The source region for excitatory inputs in the subplate is relatively small $(<200 \times$ $200 \mu \mathrm{m}$ ). Comparing the activation region in subplate (Fig. $6 B, C$ ) with projection regions in layer 4 (Fig. $6 D 3$ ) suggests that each layer 4 neuron receives input from 2 to 3 SPNs. The latency of the pEPSCs (Fig. 6D3, bottom) is similar to those of SPN spiking (Fig. $6 C$ ). The results of LSPS of a layer 4 neurons at P13 are shown in Figure 6E. In addition to subplate inputs (13/21
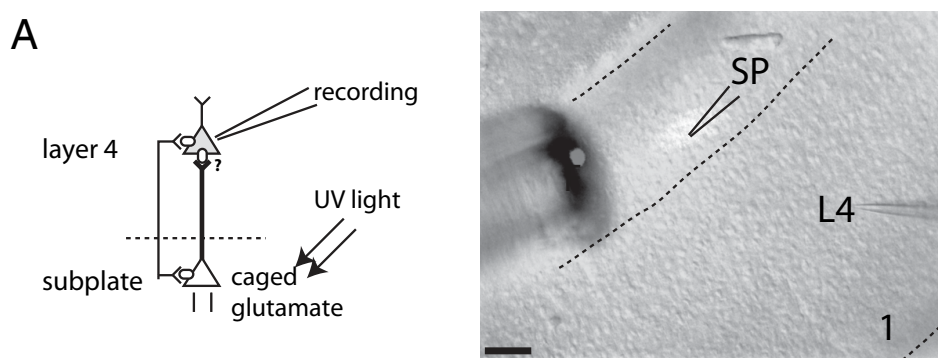

\section{Subplate recording, stimulation in subplate}
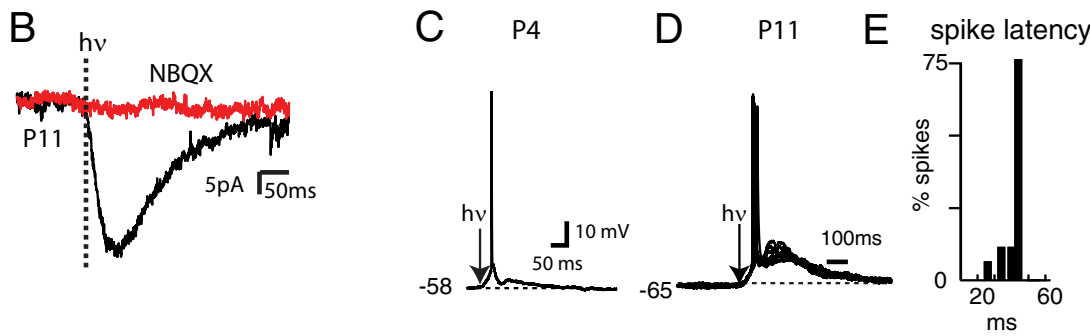

\section{Layer 4 recording, stimulation in subplate}
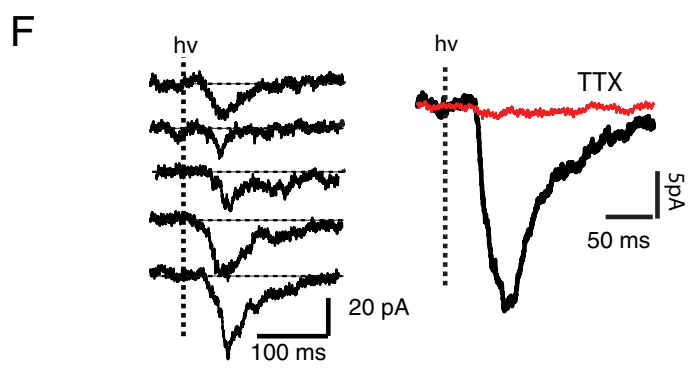

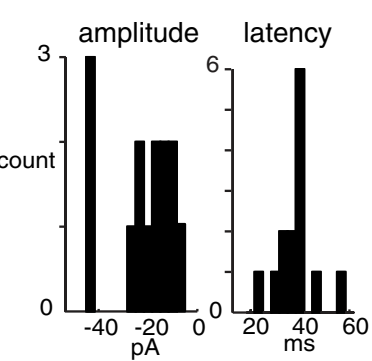

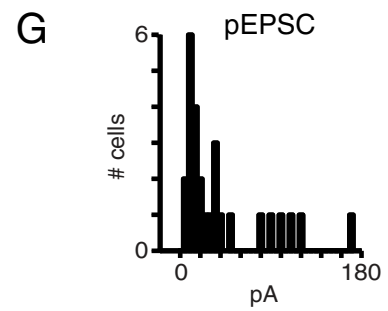

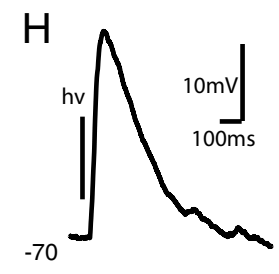

Figure 5. SPNs excite layer 4 neurons. $\boldsymbol{A}$, Left, Schematic of experimental setup. Recordings are obtained from layer 4 neurons while SPNs are excited by photostimulation. Right, Actual setup of photostimulation experiment. Image shows part of the thalamocortical slice at P4. Layer 1 is to the right (1) and white matter (WM) is to the left. Recording of layer 4 neuron through whole-cell pipette (P) while photostimulation fiber (F) and light spot (white) are located in subplate. Note that the elliptical uncaging spot does not extend into overlying cortical plate. The shape of the spot depends on the size of the fiber, its angle and distance to the slice. Imaging artifacts surrounding the fiber are due to DIC illumination. Scale bar, $100 \mu \mathrm{m} . \boldsymbol{B}-\boldsymbol{D}$, Recordings from SPNs. $\boldsymbol{B}$, Photostimulation of SPN at P11. Following a laser pulse $(\mathrm{h} \nu$ ), a rapid inward current develops (black trace) that is blocked by NBQX ( $50 \mu \mathrm{m}$; red trace). $C, D$, Photostimulation of SPNs in current clamp at P4 ( $)$ and P11 (D) reliably evokes action potentials. Occasionally 2 action potentials were observed. E, Spike latency distribution in cell from $\boldsymbol{D}$. Spike jitter is low: $75 \%$ of spikes occurred within $3 \mathrm{~ms}$ of each other during photostimulation. Absolute latency was higher in this cell due to hyperpolarized holding potential. $\boldsymbol{F}-\boldsymbol{H}$, Recordings in layer 4 cells. $\boldsymbol{F}$, Left, pEPSCs in layer 4 neurons elicited by SPN photostimulation at P11.Center, the average of 15 pEPSCs (black trace); thepEPSCswere completely blocked by TTX (0.5 $\mu$ m; red trace). Note prolonged delay after laser pulse $(\mathrm{h} \nu)$ before onset of inward current. Right, Histograms of pEPSC latency and amplitude. pEPSC latencies match those of SPN spiking $(\boldsymbol{E})$. Amplitude histogram shows 2 peaks at $\sim 20 \mathrm{pA}$ and at $\sim 40$ pA possibly indicating the summation of 2 presynaptic inputs. G, Distribution of pEPSC amplitudes in 27 layer 4 neurons. Mean amplitude was $40.6 \pm 41.2$ pA. $\boldsymbol{H}$, pEPSP in current clamp in P6 layer 4 neuron (spiking was blocked with the Na channel antagonist, QX314, in the pipette).

cells), layer 4 neurons receive inputs locally from layer 4 , from layer $2 / 3$, and in some cases projections from layer 6 (9/21 cells) (Fig. 6, compare $D, E$ ).

In the adult layer 6 , neurons can send some projections into layer 4 , even though this projection might be relatively weak in auditory cortex (Barbour and Callaway, 2008). Since layer 6 neurons can send dendrites into the subplate it is possible that our 


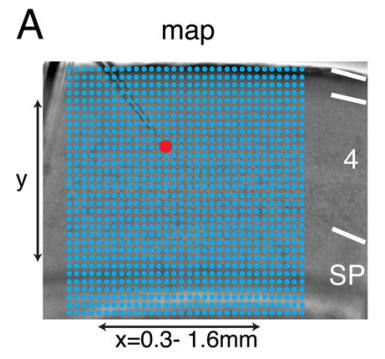

D1 Layer 4
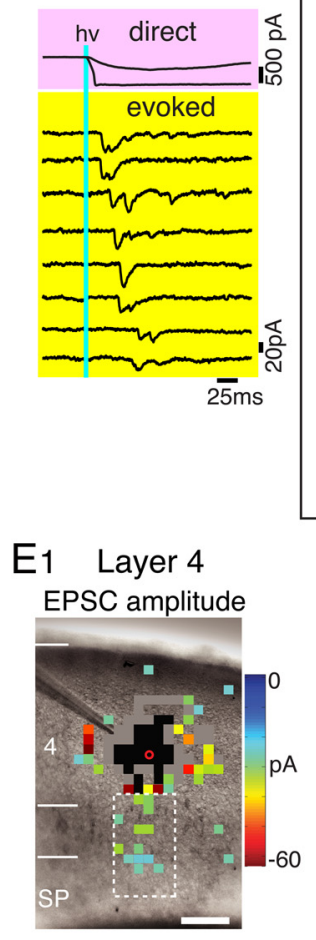

B SPN

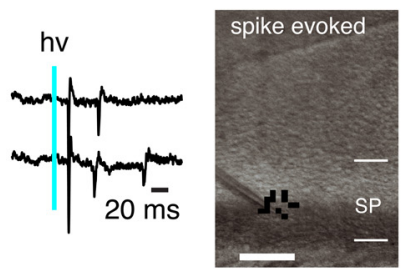

$\mathrm{C}_{1}$ SPN $\mathrm{C}_{2}$

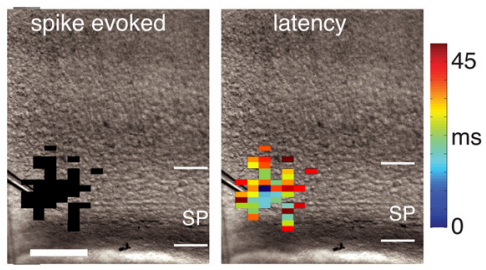

D3 EPSC amplitude

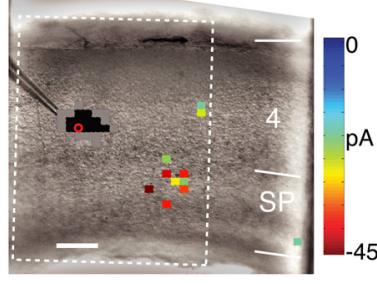

EPSC latency

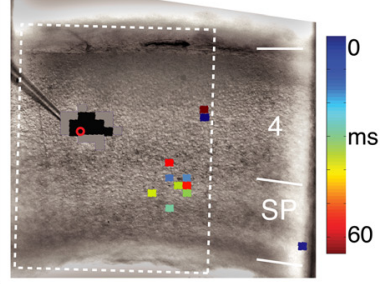

$100 \mathrm{~ms}$ 응

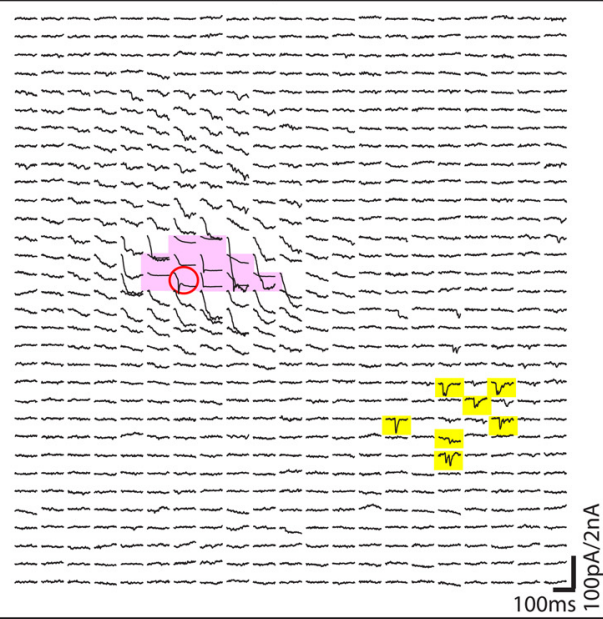

E2

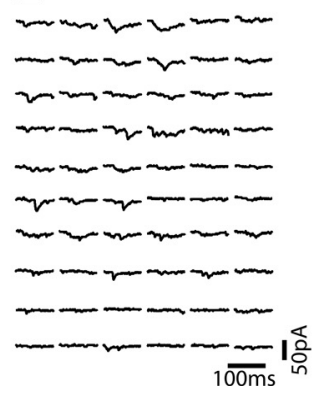

$\mathrm{F}$

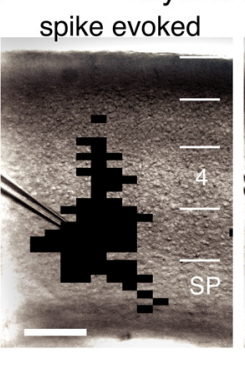

latency

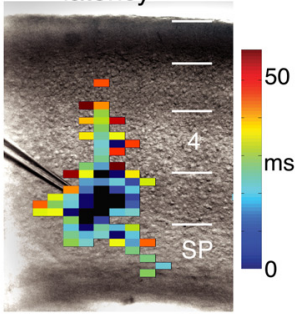

Figure 6. SPN excitation to layer 4 is focal. $A$, LSPS stimulation grid ( $32 \times 32$ spots, $50 \mu \mathrm{m}$ spacing) superimposed on infrared image. Red dot indicates cell body of recorded neuron, in this case a layer 4 neuron. $\boldsymbol{B}$, Cell-attached recording from SPN at P13. Traces show action potentials that are evoked in SPN during LSPS. To the right is a DIC image showing the recording pipette. The layer 6/subplate border is identified by large pyramidal cells in layer 6 and horizontal SPNs in upper subplate. The stimulation map is superimposed on the DIC image. Areas that caused action potentials are labeled black. Note that photostimulation can evoke multiple spikes and that region of activation is very small. C, Cell attached recording from a SPN at P9. Image in C1 shows areas that cause an action potential in black. The layer 6/subplate border is identified by large pyramidal cells in layer 6 and horizontal SPNs in upper subplate. Note that this are as confined to subplate. Image in C2 shows, for the same cell as in C1, the latency to first spike of cell in C1 encoded in pseudocolor. Areas closer to the cell body show shorter latencies. D, Whole-cell patch recordings of layer neuron (P7) during LSPS.D1, Photostimulation (blue line) evoked short latency and long latency EPSCs labeled direct and evoked. D2, Traces show poststimulation currents (100 ms) at stimulus locations for this

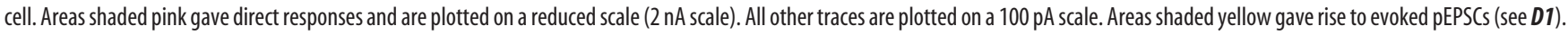
Note the large gap between these two areas. pEPSCs originating from superficial locations partially overlap direct response. D3, pEPSC amplitude (top) and latency (bottom) as function of stimulus xy-position and superimposed on DIC image of slice. Values are encoded in pseudocolor. Locations that led to short latency direct responses are marked in black. Red circle indicates location of cell body. White box indicates stimulated area. E, LSPS of a layer 4 neuron at P13. E1 shows amplitude map. In addition to SPN input, this neuron also received intracortical inputs from layers 2/3 and 4. E2, Traces show poststimulation currents during stimulation of areas overlying layer 6 and subplate (white box in E1). Note discontinuity of stimulation sites in layer 6 that gave responses from those in subplate that gave responses suggesting that these stimulation sites activate distinct populations of cells. $F$, Cell attached recording from layer 6 neuron. Left image shows areas that cause an action potential. Note that this area is large and includes a wide area in layer 6 and a narrow area in layer 4 . Right image shows latency to first spike encoded in pseudocolor. Note the short latency to first spike for somatic stimulation. All scale bars, $200 \mu \mathrm{m}$.

stimulation activates layer 6 neurons. To investigate if layer 6 neuron activation can contribute to the observed EPSCs in layer 4 neurons, we mapped directly the activation of layer 6 neurons $(N=2)$ and found that layer 6 neurons were readily excited by stimulation in a large area surrounding their soma but also in an area extending radially toward the pia (Fig. $6 F$ ). The latter area likely coincides with the prominent apical dendrite of layer 6 pyramidal cells (Fig. $1 D$ ). This large activation area is similar to those reported in layer 2/3 and layer 5 pyramidal cells (Shepherd et al., 2003). Thus, layer 6 neurons can be activated from a much larger area than SPNs. The large activation area of layer 6 neurons is larger than the size of the source of excitatory connections to layer 4 neurons. In particular, the region that gives rise to EPSCs in layer 4 (Fig. $6 D, E$ ) does not include the large activation regions of layer 6 cells (Fig. $6 F$ ), suggesting that there is a excitatory connection between SPNs and layer 4 neurons. 


\section{Discussion}

Our data show that SPNs are a strong excitatory relay of thalamic information to the developing cortical layer 4 before and after the onset of hearing. We also show that SPNs provide strong excitatory input to layer 4 .

We report that SPNs receive MGN inputs and that these inputs are strong enough to trigger action potentials in SPNs. This strong effect of relatively weak synaptic inputs may be mediated by the high input resistances of SPNs. Thus, in effect, the "immature" intrinsic characteristics of SPNs (high Rm) might compensate for the immature synaptic inputs (small amplitudes). High input resistances in early development are not unique to SPNs: for example, layer $2 / 3$ cells show a similar progression $\sim 10 \mathrm{~d}$ later (Oswald and Reyes, 2008). The decrease in input resistance and resting potential of SPNs over development are consistent with the increased expression of K-channels during the postnatal period. Importantly, the functional compensation of weak immature synapses by coincident development of intrinsic mechanisms points to a general mechanism whereby neural circuits can be functional even though their components have not matured. One feature of immature SPNs is that the action potential latency is very short, allowing SPN firing to preserve the timing information present in the thalamic inputs. Such preservation of timing information has been shown to be important in computational models of the role of SPNs in cortical development (Kanold and Shatz, 2006).

While we observed a strengthening of the maximal MGN EPSC over development, we also observed a large increase in variance in EPSC amplitudes (Fig. 2C). We interpret this variability as originating from our preparation, since growth over development prevents us from preserving the entire thalamocortical pathway in a slice preparation at older ages. It seems likely that the maximal observed EPSC at older ages represents a lower estimate of the true total MGN input onto SPNs.

Thalamic input has been implicated in the survival of SPNs (Price and Lotto, 1996) in that removal of thalamic innervation from SPNs after their ingrowth and strengthening in layer 4 would cause the death of SPNs by depriving them of their excitatory inputs. However, we observe an increase rather than a decrease in the number of MGN inputs to SPNs during a period when thalamic inputs mature. Thus, retraction of thalamic inputs is unlikely to be the "death signal" for SPN neurons. Imaging experiments show that in S1 thalamic activation of subplate seems to decline by P10 (Higashi et al., 2005). This is consistent with our observation of a decrease in input resistance and thus excitability, which can counteract increases in synapse number. Thus, SPN death could be caused by reduced functional activation of SPNs.

In some areas of the brain synaptic circuits refine by initial exuberant growth and later pruning, while in other areas the numbers of synaptic inputs increase without evidence of hyperinnervation (LeVay et al., 1978; Bureau et al., 2004; Chen and Regehr, 2000; Friedlander and Martin, 1989; Price and Blakemore, 1985). We here find that thalamic synapses onto SPNs strengthen over development. However, compared to other areas of the brain there are distinct differences in SPN circuits, in that the majority of SPNs die. Thus, it might be that remaining SPNs "inherit" MGN inputs from SPNs targeted for programmed cell death and that SPNs at older ages showing smaller MGN input are the ones targeted for "cell death." Such a mixed population of SPNs could also contribute to the large variability of maximal EPSCs seen in our data (Fig. 2C).

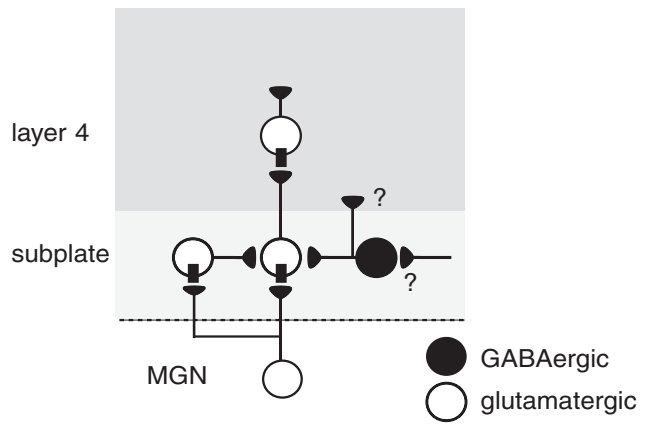

Figure 7. Model circuit of SPNs in auditory cortex. Excitatory SPNs receive input from MGN, while inhibitory SPNs do not receive inputs from MGN but from other unknown sources ("?"). Excitatory SPNs project to layer 4 and to other SPNs, while the projection targets of inhibitory SPNs are unknown.

Following MGN stimulation, we occasionally observed disynaptic EPSCs in SPNs, especially with higher stimulation intensities. Thus, MGN stimulation likely activates excitatory SPNs, which appear to synapse on other SPNs (Fig. 7). Electrical stimulation of the white matter (which activates SPNs) activates GABAergic inputs on SPNs, thus it is likely that GABAergic SPNs innervate other SPNs (Voigt et al., 2001; Hanganu et al., 2002). However, we did not observe any IPSCs after MGN stimulation, even when holding SPNs at depolarized potentials. Thus, GABAergic SPNs might not be strongly driven by MGN inputs, but might be driven by input from other sources (Fig. 7).

SPN axons project to and ramify in cortical layer 4 (Friauf et al., 1990; Allendoerfer and Shatz, 1994; Piñon et al., 2009), but a functional connection between SPNs and layer 4 had not been directly demonstrated. Since the large majority of SPN that project to layer 4 are found to be glutamatergic (Finney et al., 1998), an excitatory connection had been hypothesized. Our results here show directly that SPNs provide strong excitatory inputs to layer 4 neurons throughout the postnatal period. While in many cortical regions in the adult layer 6 neurons project to layer 4 (Douglas and Martin, 2004), our data suggests that such projections might be weak or sparse at early ages in the auditory cortex. This is consistent with data in ferret visual cortex showing a late development of the axonal projection from layer 6 to layer 4 (Callaway and Lieber, 1996). In addition, recent results suggest that in auditory cortex the strength of layer 6 to layer 4 projections might be low (Barbour and Callaway, 2008), consistent with our findings. Our observation of projections from layer $2 / 3$ to layer 4 in ACX confirms recent observation in older animals using similar techniques (Barbour and Callaway, 2008).

In the adult, remaining subplate neurons (layer $6 \mathrm{~b}$ ) provide input to cortical layer 6 (Torres-Reveron and Friedlander, 2007). This is consistent with our data showing that at older ages subplate activity might drive cortical circuits that excite layer 6 neurons (Fig. 3). Together, our data indicates that the developing cortical circuit operates in a feedforward and not recurrent manner. This is consistent with results in the developing barrel cortex (Bureau et al., 2004) showing early columnar pattern of connections. The presence of long latency activity in layer 6 following MGN stimulation is also consistent with the view that SPNs act as a generator or amplifier of cortical oscillatory activity (Dupont et al., 2006; Hanganu et al., 2009).

Our LSPS data show that layer 4 neurons receive SPN input from locations in the superficial subplate. Many of these superficial SPNs survive and form layer 6B (Torres-Reveron and Friedlander, 2007) while many of the deeper SPNs disappear, 
except for some sometimes termed "white matter neurons" (Torres-Reveron and Friedlander, 2007). Thus, the subplate may contain multiple circuits of which one projects to layer 4 . Recent advances in molecularly typing SPNs (HoerderSuabedissen et al., 2009; Osheroff and Hatten, 2009) might be a tool to allow the functional dissection of these different circuits.

Cell culture studies had suggested that GABAergic SPNs play a role in organizing cortical activity (Voigt et al., 2001), but we did not find evidence of GABAergic inputs from SPNs to layer 4 in our recordings. Thus, GABAergic SPNs might locally organize the activity of excitatory SPNs, or project outside of layer 4 . We did not observe MGN-driven inhibition in SPNs, suggesting that MGN inputs do not drive GABAergic SPNs. Since we did not identify the transmitter phenotype of the recorded SPNs it is possible that some recorded SPNs are GABAergic. In fact we occasionally recorded SPNs that did not receive MGN input. While the lack of MGN input could be due to slicing artifacts, it is also possible that we recorded from GABAergic SPN that did not receive MGN inputs. However, together with the absence of MGN-driven inhibition in SPNs, it can be proposed that there are distinct parallel circuits within the subplate, one providing feedforward excitatory connections as studied here, and another containing GABAergic neurons.

The excitatory projection from SPNs to layer 4 neurons forms a substrate whereby SPNs can relay or influence early spontaneous activity in cortex (Dupont et al., 2006). While SPNs can also be coupled to other SPNs via electrical synapses (Dupont et al., 2006), we did not see evidence of electrical coupling to layer 4 neurons in experiments in which spiking was blocked by TTX (Fig. 5F). Gap junction coupling would have been revealed by short-latency, TTX-resistant depolarization or inward current, which we did not observe. However, it is possible that electrical synapses between SPNs and other cortical neurons outside of layer 4 exist. It might also be the case that gap junction coupling might exert a local effect on dendritic processing that we cannot observe via somatic whole-cell recordings from layer 4 neurons due to insufficient voltage clamp. Figure 7 summarizes our current view of the SPN associated circuit in A1.

Together, results here show directly that SPNs provide strong excitatory inputs to layer 4 neurons throughout the postnatal period. This excitatory projection can interact with learning rules present at layer 4 synapses to enable strengthening of thalamocortical synapses and plasticity during the critical period (Kanold et al., 2003; Kanold and Shatz, 2006). For example, by depolarizing layer 4 neurons, SPNs can enhance mechanisms of heterosynaptic LTP at the thalamocortical synapse or increase correlations between thalamic and cortical activity so that the thalamocortical synapse can be strengthened via spike-timing-dependent plasticity mechanisms (Kanold and Shatz, 2006).

Our results show that SPNs are tightly integrated in the developing thalamocortical circuit. SPNs receive and relay thalamic information to the developing cortical layer 4 . Via these feedforward excitatory connections, SPNs may control activity levels and patterns in layer 4 that ultimately lead to the functional maturation of layer 4. Loss of this excitatory feedforward circuit caused by injury in development (McQuillen and Ferriero, 2005) could prevent activity-dependent maturational processes and leave layer 4 in an immature state, as has been observed experimentally following SPN ablation (Kanold et al., 2003; Kanold and Shatz, 2006). Thus, SPNs form a key circuit during cortical development, tightly linking the thalamus to the developing cortical layer 4 and enabling thalamocortical development.

\section{References}

Alford BR, Ruben RJ (1963) Physiological, behavioral and anatomical correlates of the development of hearing in the mouse. Ann Otol Rhinol Laryngol 72:237-247.

Allendoerfer KL, Shatz CJ (1994) The subplate, a transient neocortical structure: its role in the development of connections between thalamus and cortex. Annu Rev Neurosci 17:185-218.

Barbour DL, Callaway EM (2008) Excitatory local connections of superficial neurons in rat auditory cortex. J Neurosci 28:11174-11185.

Bureau I, Shepherd GM, Svoboda K (2004) Precise development of functional and anatomical columns in the neocortex. Neuron 42:789-801.

Callaway EM, Lieber JL (1996) Development of axonal arbors of layer 6 pyramidal neurons in ferret primary visual cortex. J Comp Neurol 376:295-305.

Catalano SM, Robertson RT, Killackey HP (1991) Early ingrowth of thalamocortical afferents to the neocortex of the prenatal rat. Proc Natl Acad Sci U S A 88:2999-3003.

Chen C, Regehr WG (2000) Developmental remodeling of the retinogeniculate synapse. Neuron 28:955-966.

Clancy B, Silva-Filho M, Friedlander MJ (2001) Structure and projections of white matter neurons in the postnatal rat visual cortex. J Comp Neurol 434:233-252.

Cruikshank SJ, Rose HJ, Metherate R (2002) Auditory thalamocortical synaptic transmission in vitro. J Neurophysiol 87:361-384.

de Villers-Sidani E, Chang EF, Bao S, Merzenich MM (2007) Critical period window for spectral tuning defined in the primary auditory cortex (A1) in the rat. J Neurosci 27:180-189.

Douglas RJ, Martin KA (2004) Neuronal circuits of the neocortex. Annu Rev Neurosci 27:419-451.

Dupont E, Hanganu IL, Kilb W, Hirsch S, Luhmann HJ (2006) Rapid developmental switch in the mechanisms driving early cortical columnar networks. Nature 439:79-83.

Ehret G, Romand R (1992) Development of tone response thresholds, latencies and tuning in the mouse inferior colliculus. Brain Res Dev Brain Res $67: 317-326$.

Finney EM, Stone JR, Shatz CJ (1998) Major glutamatergic projection from subplate into visual cortex during development. J Comp Neurol 398:105-118.

Friauf E, Shatz CJ (1991) Changing patterns of synaptic input to subplate and cortical plate during development of visual cortex. J Neurophysiol 66:2059-2071.

Friauf E, McConnell SK, Shatz CJ (1990) Functional synaptic circuits in the subplate during fetal and early postnatal development of cat visual cortex. J Neurosci 10:2601-2613.

Friedlander MJ, Martin KA (1989) Development of Y-axon innervation of cortical area 18 in the cat. J Physiol 416:183-213.

Ghosh A, Shatz CJ (1992) Involvement of subplate neurons in the formation of ocular dominance columns. Science 255:1441-1443.

Gurung B, Fritzsch B (2004) Time course of embryonic midbrain and thalamic auditory connection development in mice as revealed by carbocyanine dye tracing. J Comp Neurol 479:309-327.

Hanganu IL, Kilb W, Luhmann HJ (2002) Functional synaptic projections onto subplate neurons in neonatal rat somatosensory cortex. J Neurosci 22:7165-7176.

Hanganu IL, Okabe A, Lessmann V, Luhmann HJ (2009) Cellular mechanisms of subplate-driven and cholinergic input-dependent network activity in the neonatal rat somatosensory cortex. Cereb Cortex 19:89-105.

Higashi S, Molnár Z, Kurotani T, Toyama K (2002) Prenatal development of neural excitation in rat thalamocortical projections studied by optical recording. Neuroscience 115:1231-1246.

Higashi S, Hioki K, Kurotani T, Kasim N, Molnár Z (2005) Functional thalamocortical synapse reorganization from subplate to layer IV during postnatal development in the reeler-like mutant rat (shaking rat Kawasaki). J Neurosci 25:1395-1406.

Hoerder-Suabedissen A, Wang WZ, Lee S, Davies KE, Goffinet AM, Rakić S, Parnavelas J, Reim K, Nicolić M, Paulsen O, Molnár Z (2009) Novel markers reveal subpopulations of subplate neurons in the murine cerebral cortex. Cereb Cortex 19:1738-1750.

Kanold PO (2004) Transient microcircuits formed by subplate neurons and their role in functional development of thalamocortical connections. Neuroreport 15:2149-2153. 
Kanold PO (2009) Subplate neurons: crucial regulators of cortical development and plasticity. Front Neuroanat 3:16.

Kanold PO, Shatz CJ (2006) Subplate neurons regulate maturation of cortical inhibition and outcome of ocular dominance plasticity. Neuron 51:627-638.

Kanold PO, Kara P, Reid RC, Shatz CJ (2003) Role of subplate neurons in functional maturation of visual cortical columns. Science 301:521-525.

Kao JPY (2006) Caged molecules: principles and practical considerations. In: Current protocols in neuroscience (Gerfen C, Holmes A, Rogawski M, Sibley D, Skolnick P, Wray S, eds), pp 6.20.1-6.20.21. Hoboken, NJ: Wiley.

LeVay S, Stryker MP, Shatz CJ (1978) Ocular dominance columns and their development in layer IV of the cat's visual cortex: a quantitative study. J Comp Neurol 179:223-244.

Luhmann HJ, Reiprich RA, Hanganu I, Kilb W (2000) Cellular physiology of the neonatal rat cerebral cortex: intrinsic membrane properties, sodium and calcium currents. J Neurosci Res 62:574-584.

McQuillen PS, Ferriero DM (2005) Perinatal subplate neuron injury: implications for cortical development and plasticity. Brain Pathol 15:250-260.

Mikaelian D, Ruben RJ (1965) Development of hearing in the normal Cba-J mouse: correlation of physiological observations with behavioral responses and with cochlear anatomy. Acta Otolaryngol 59:451-461.

Mikaelian D, Alford BR, Ruben RJ (1965) Cochlear potentials and 8 nerve action potentials in normal and genetically deaf mice. Ann Otol Rhinol Laryngol 74:146-157.

Molnár Z, Blakemore C (1995) Guidance of thalamocortical innervation. Ciba Found Symp 193:127-149; discussion 192-199.

Molnár Z, Kurotani T, Higashi S, Yamamoto N, Toyama K (2003) Development of functional thalamocortical synapses studied with current source-density analysis in whole forebrain slices in the rat. Brain Res Bull 60:355-371.

Osheroff H, Hatten ME (2009) Gene expression profiling of preplate neurons destined for the subplate: genes involved in transcription, axon extension, neurotransmitter regulation, steroid hormone signaling, and neuronal survival. Cereb Cortex 19 [Suppl 1]:i126-i134.

Oswald AM, Reyes AD (2008) Maturation of intrinsic and synaptic properties of layer $2 / 3$ pyramidal neurons in mouse auditory cortex. J Neurophysiol 99:2998-3008.

Piñon MC, Jethwa A, Jacobs E, Campagnoni A, Molnár Z (2009) Dynamic integration of subplate neurons into the cortical barrel field circuitry during postnatal development in the Golli-tau-eGFP (GTE) mouse. J Physiol 587:1903-1915.

Price DJ, Blakemore C (1985) Regressive events in the postnatal development of association projections in the visual cortex. Nature 316:721-724.

Price DJ, Lotto RB (1996) Influences of the thalamus on the survival of subplate and cortical plate cells in cultured embryonic mouse brain. J Neurosci 16:3247-3255.

Price DJ, Aslam S, Tasker L, Gillies K (1997) Fates of the earliest generated cells in the developing murine neocortex. J Comp Neurol 377:414-422.

Pujol R, Lavigne-Rebillard M, Lenoir M (1997) Development of sensory and neural structures in the mammalian cochlea. In: Development of the auditory system (Rubel E, Popper A, Fay R, eds), pp 146-192. Berlin: Spinger.

Reep RL (2000) Cortical layer VII and persistent subplate cells in mammalian brains. Brain Behav Evol 56:212-234.

Romand R, Ehret G (1990) Development of tonotopy in the inferior colliculus. I. Electrophysiological mapping in house mice. Brain Res Dev Brain Res 54:221-234.

Ruebsamen R, Lippe W (1997) The development of cochlear function. In: Development of the auditory system (Rubel E, Popper A, Fay R, eds), pp 193-270. Berlin: Springer.

Shepherd GM, Pologruto TA, Svoboda K (2003) Circuit analysis of experience-dependent plasticity in the developing rat barrel cortex. Neuron 38:277-289.

Shnerson A, Pujol R (1983) Development: anatomy electrophysiology and behavior. In: The auditory psychobiology of the mouse (Willot JF, ed), pp 395-425. Springfield IL: Charles C Thomas.

Shnerson A, Lenoir M, van de Water TR, Pujol R (1983) The pattern of sensorineural degeneration in the cochlea of the deaf shaker-1 mouse: ultrastructural observations. Brain Res 285:305-315.

Torres-Reveron J, Friedlander MJ (2007) Properties of persistent postnatal cortical subplate neurons. J Neurosci 27:9962-9974.

Vandevelde IL, Duckworth E, Reep RL (1996) Layer VII and the gray matter trajectories of corticocortical axons in rats. Anat Embryol 194:581-593.

Voigt T, Opitz T, de Lima AD (2001) Synchronous oscillatory activity in immature cortical network is driven by GABAergic preplate neurons. J Neurosci 21:8895-8905. 